\title{
Recent Developments and Applications on Qualitative Theory of Fractional Equations and Related Topics
}

\author{
Shurong Sun, ${ }^{1}$ Luisa Morgado, ${ }^{2}$ Jehad Alzabut, ${ }^{3}$ and Ivanka Stamova ${ }^{4}$ \\ ${ }^{1}$ School of Mathematical Sciences, University of Jinan, Jinan, Shandong 250022, China \\ ${ }^{2}$ Mathematics Department, School of Science and Technology, University of Trás-os-Montes e Alto Douro, Vila Real, Portugal \\ ${ }^{3}$ Department of Mathematics and Physical Sciences, Prince Sultan University, P.O. Box 66833, Riyadh 11586, Saudi Arabia \\ ${ }^{4}$ Department of Mathematics, University of Texas at San Antonio, San Antonio, TX 78249, USA
}

Correspondence should be addressed to Shurong Sun; sshrong@163.com

Received 7 December 2014; Accepted 7 December 2014

Copyright (C) 2015 Shurong Sun et al. This is an open access article distributed under the Creative Commons Attribution License, which permits unrestricted use, distribution, and reproduction in any medium, provided the original work is properly cited.

Recently, fractional differential equations have been the object of considerable interest amongst researchers. This is justified by the intensive development of the theory of fractional differential equations itself and by the widespread applications of such construction in various sciences such as physics, mechanics, chemistry, and engineering. On the other hand, it is well known that the qualitative theory of differential equations can be very useful in applications. Much attention has been given to the notions of stability, oscillation, asymptotic, existence, and uniqueness theory of differential equations over the past decades. The explosion in research within the fractional differential equation settings has led to new developments in qualitative theory of fractional differential equations. Indeed, it is well known that the analysis of fractional differential equations is more complex than that of classical differential equations, since fractional derivatives are nonlocal and have weakly singular kernels. Therefore, the development of qualitative theory, especially oscillation, of nonlinear fractional differential equations has been slowly developed a bit slow.

This issue on recent developments and applications on qualitative theory of fractional equations and related topics aims to provide a wide survey on the notions of stability, existence, uniqueness, and oscillation theory of differential equations of noninteger order, on the development of numerical methods for such equations, and on the numerical simulation and convergence analysis for qualitative theory of fractional differential equations and their applications. This issue contains many fascinating papers of different disciplines, most of them focusing on the existence, uniqueness, and multiplicity of solutions, solvability, stability, and oscillation for fractional differential equations and discrete fractional equations.

The existence, uniqueness, and multiplicity of solutions are considered to be fundamental in the study of fractional differential equations. Y. Cui and Y. Zou discuss the existence of extremal solutions for nonlinear fractional differential systems with coupled four-point boundary value problems by establishing a comparison result and using the monotone iterative technique combined with the method of upper and lower solutions. L. Wang et al. investigate the existence of positive solutions for a class of singular $\mathrm{p}$-Laplacian fractional differential equations with integral boundary conditions by using the Leggett-Williams fixed point theorem and obtained some sufficient conditions for the existence of at least three positive solutions. D. Yang studies the existence of solutions of second-order three-point boundary value problems with impulse by constructing a variational functional of the boundary value problem and using the critical point theory. M. Yang and A. Li, concerned with the multiplicity of solutions to elliptic equations with combined nonlinearities and parameter, discuss two sufficient conditions for multiple nontrivial radial solutions in terms of the range of the parameter. W. Jian and H. Sun consider eigenvalues of complex Sturm-Liouville boundary value problems. Lower bounds on the real parts of all eigenvalues are given in terms of the coefficients of the corresponding equation and the bound on the imaginary part of each eigenvalue is obtained in terms 
of the coefficients of this equation and the real part of the eigenvalue.

Numerical methods of differential equations are used to find approximate solutions whenever the analytical ones are not available, which is usually the case of fractional differential equations. Several methods for the approximate solutions to classical differential equations are extended to solving differential equations of fractional order numerically. H. Ding et al. develop high-order numerical algorithms for the left and right Riemann-Liouville derivatives, and, using the derived schemes, they get high-order approximations for the Riesz fractional derivative. Based on the approximate algorithm, a numerical scheme for the space Riesz fractional diffusion equation is constructed, where a compact difference scheme is applied to approximate the first-order time derivative. The stability and the convergence of the obtained finite difference scheme is discussed. J. Liu et al. introduce a new Dadras system with complex variables which can exhibit both fourwing hyperchaotic and chaotic attractors. Some dynamic properties of the system have been described including Lyapunov exponents, fractal dimensions, and Poincaré maps. Numerical results verify the feasibility and effectiveness of the presented schemes.

The continuous fractional differential equation has seen tremendous growth within the last ten years or so. The discrete fractional equation, on the other hand, has seen slower progress. In the last years, however, a number of papers have appeared, and these have begun to build up the theoretical foundations of the discrete fractional equation. $\mathrm{W}$. Lv studies the existence of solutions to discrete threepoint boundary value problem at resonance involving the Riemann-Liouville fractional difference; the existence result is established by using the coincidence degree continuation theorem. K. Cheng et al. establish some new weakly singular versions for discrete nonlinear inequalities and apply them to discuss the upper bound and the uniqueness of solutions of a Volterra type difference equation with certain weakly singular kernels.

Stability and oscillation are important issues of differential equations. F. Qiu and Q. Zhang investigate the robust delay-dependent stability problem for neutral system with mixed delays and nonlinear perturbations. A delay decomposition approach is used so that information of the delayed plant states can be taken into full consideration. Based on a special Lyapunov functional approach, a novel delaydependent stability criteria are obtained in terms of linear matrix inequalities (LMIs). A numerical example illustrates the effectiveness of the derived method and the improvement over some existing methods. S. Xiang et al. investigated the oscillation for a class of fractional differential equation with Liouville derivative. Using a generalized Riccati function and inequality technique they establish some new oscillation criteria. Q. Zhang and X. Song discuss oscillation criteria for second-order half-linear neutral delay dynamic equations on time scales by using the generalized Riccati transformation and some inequality techniques. Circulant type matrices have been exploited to obtain the transient solution in a closed form for fractional differential equations and used to get the Routh-Hurwitz stability conditions from the eigenvalues of circulant matrices for fractional differential systems. Y. Gong et al. consider the circulant, left circulant and gcirculant matrices with the Jacobsthal and Jacobsthal-Lucas numbers. They discuss the invertibility of the circulant matrix and present the determinant and the inverse matrix. The invertibility of the left circulant and g-circulant matrices is also discussed.

Special functions, such as Mittag-Leffler function and Gauss Hypergeometric function, play an important role in the determination of analytical solutions to fractional order differential or integral equations. Their importance is due to their direct involvement in the problems of physics, biology, engineering, and applied sciences. Several extensions of the well-known special functions like beta function, gamma functions, and Mittag-Leffler and Gauss hypergeometric functions and their properties started to be one of the main directions for researchers. A remarkably large number of fractional integral formulas involving the number of special functions have been investigated. D. Baleanu and P. Agarwal establish image formulas for the generalized Gauss hypergeometric function by applying generalized operators of the fractional integration involving Appell's function, and the results obtained in this paper are useful in deriving certain composition formulas involving various fractional integral operators and generalized Gauss hypergeometric functions.

In conclusion, we hope that the papers published in this issue will enrich the readers' knowledge and stimulate researchers to extend, generalize, and apply the established results in their future works.

\section{Acknowledgment}

We would also like to thank the editorial board members of this journal, for their support and help throughout the preparation of this special issue.

Shurong Sun
Luisa Morgado
Jehad Alzabut
Ivanka Stamova




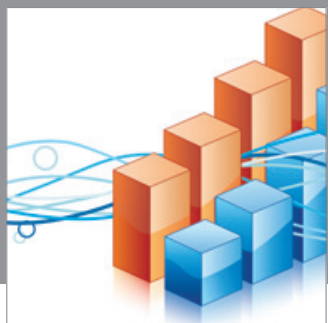

Advances in

Operations Research

mansans

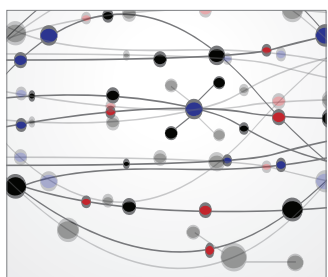

The Scientific World Journal
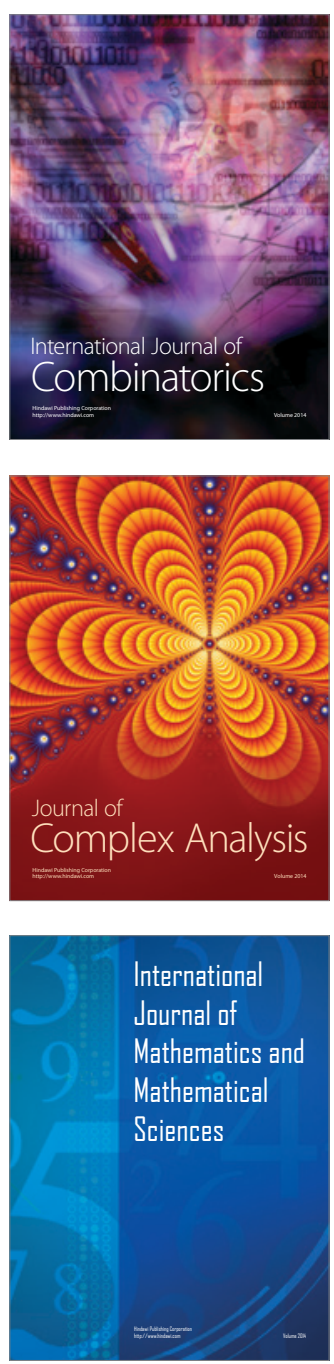
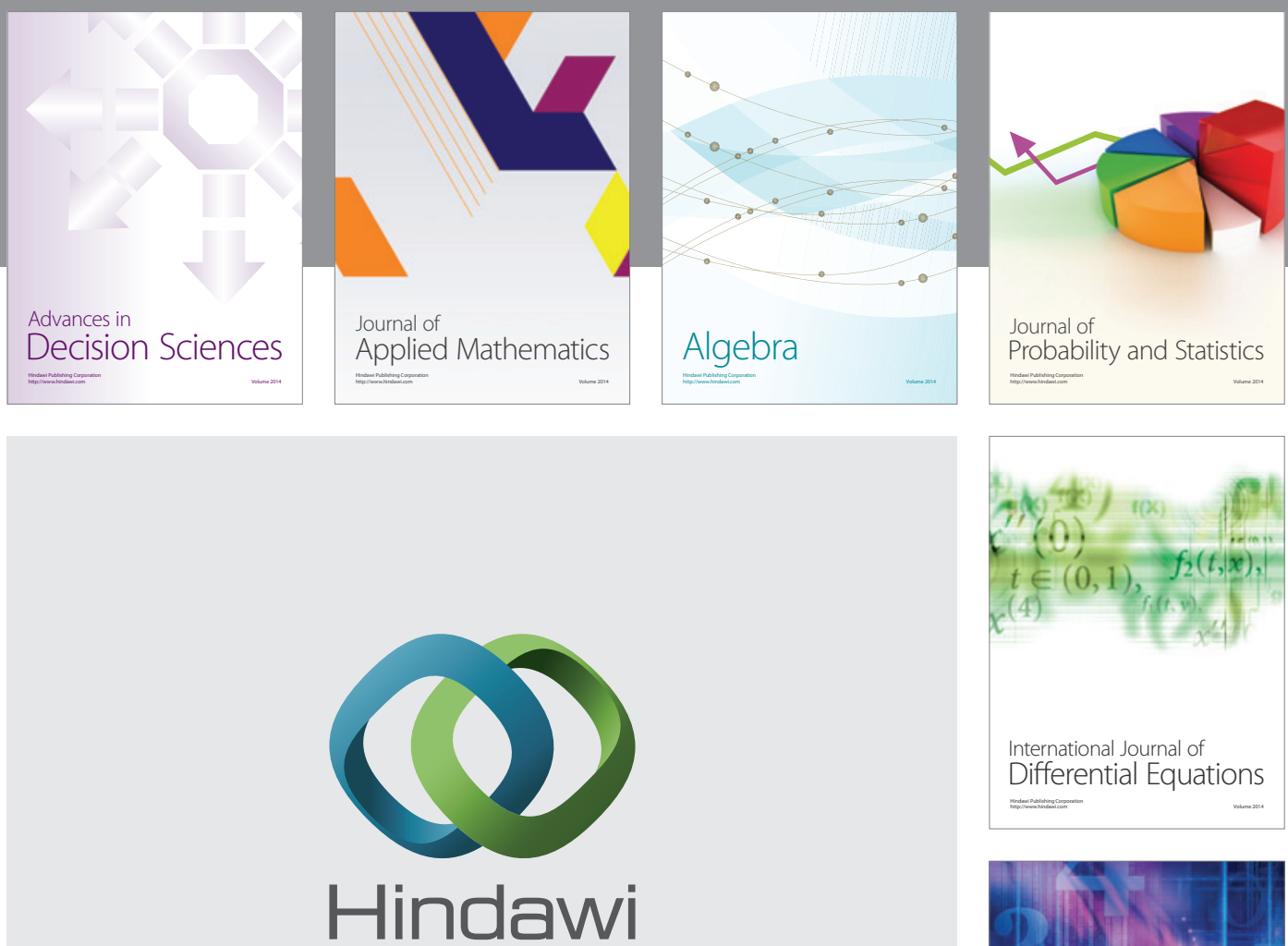

Submit your manuscripts at http://www.hindawi.com
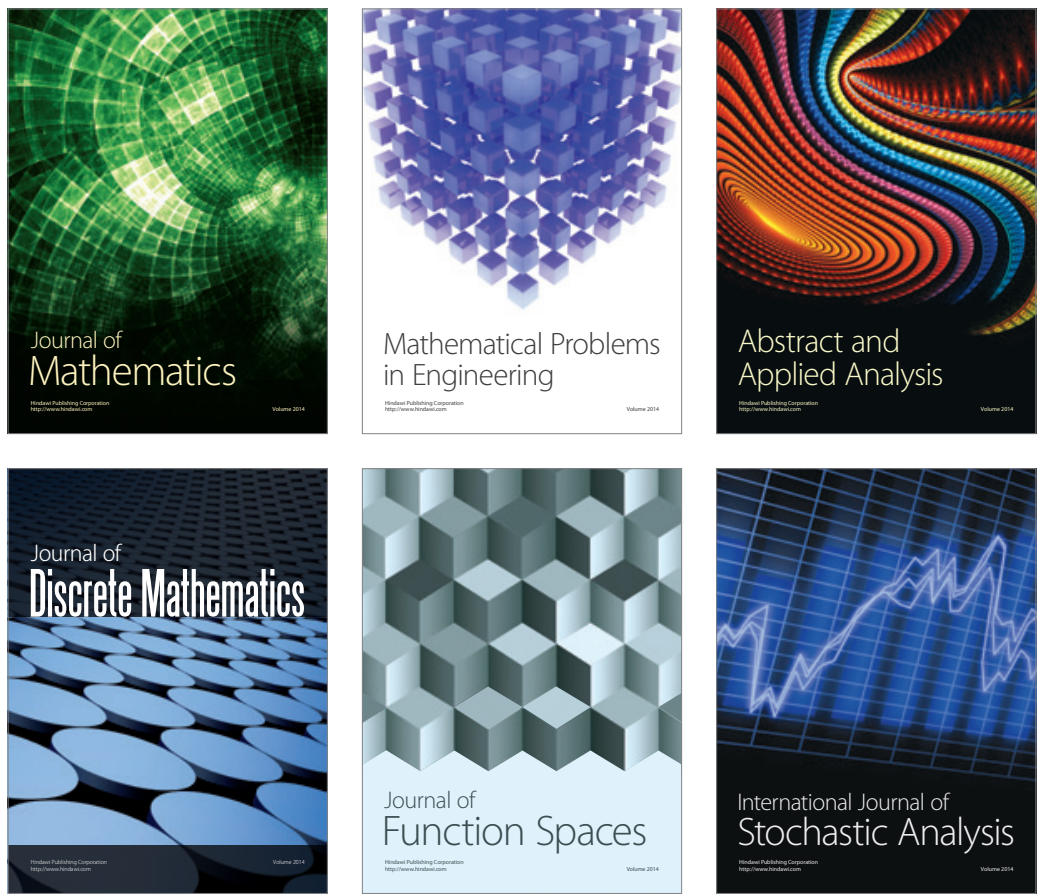

Journal of

Function Spaces

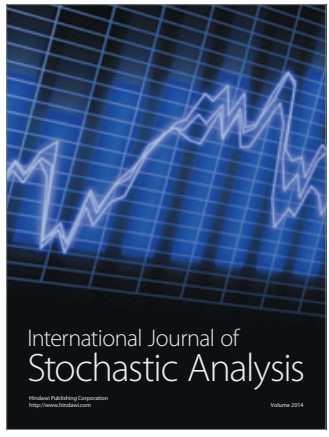

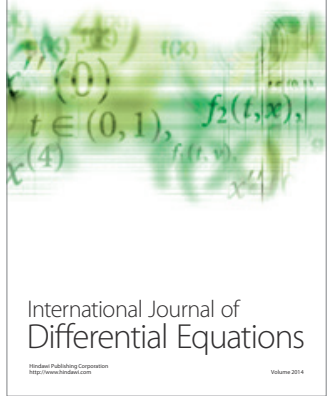
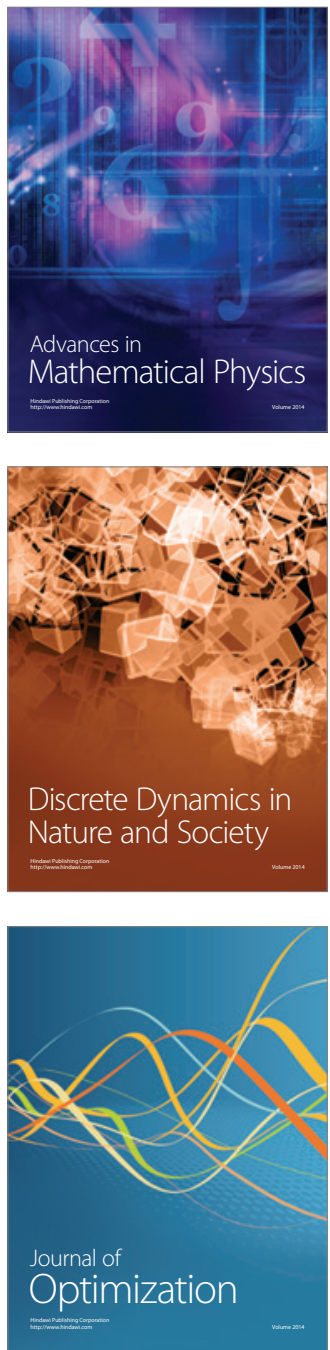